\title{
BOREL MAPS WITH THE "POINT OF CONTINUITY PROPERTY" AND COMPLETELY BOREL ADDITIVE FAMILIES IN SOME NONMETRIZABLE SPACES
}

\author{
PETR HOLICKÝ
}

(Communicated by Franklin D. Tall)

\begin{abstract}
Under the axiom that no measurable cardinal exists it is proved that " $(F \cap G)_{\sigma}$-measurable" maps of a hereditarily Baire and Čech analytic (e.g., compact) space into a metric space has the point of continuity property. A result on completely Borel-additive families in Cech analytic spaces is the crucial part of the proof.
\end{abstract}

In his lecture at the Topological Symposium in Prague 1991, Hansell asked the question if his Theorem 2.8 from [H1] holds for compact spaces $T$, i.e., if any map $f$ of $T$ to a metric space $X$ with preimages of open sets being of the form $\bigcup_{n=1}^{\infty}\left(F_{n} \cap G_{n}\right), F_{n}$ closed and $G_{n}$ open, is a $P C$-function, i.e., $f \mid A$ has a point of continuity for each nonempty closed subset $A$. Using his Theorem 2.2 from the same paper we show that it is true under the axiom

no cardinal admits a nontrivial two-valued $\sigma$-additive measure,

(A) defined on the $\sigma$-algebra of all subsets, with the measure of singletons being zero.

We first formulate the main results, then explain the notions used in them, and finally we give the proofs.

\section{RESULTS}

Theorem 1 (under (A)). If $f$ is an $(\mathscr{F} \wedge \mathscr{G})_{\sigma}$-measurable map of a hereditarily Baire and almost $K$-descriptive completely regular Hausdorff space (e.g., a product of a compact space and a complete metric space) $T$ to a metric space $X$ then $f$ is a PC function.

Remark. We recall a definition of almost $K$-descriptive spaces below. Let us point out that all Čech complete or even all Čech analytic spaces are almost $K$-descriptive [Ho, Theorem 1].

To prove Theorem 1 we use the equivalence following from Theorem 2.2 of [H1].

Received by the editors January 16, 1992.

1991 Mathematics Subject Classification. Primary 54H05, 28A05, 04 A15.

Key words and phrases. Borel map, point of continuity property, scattered family, relatively discrete family network, completely Borel-additive family, almost $K$-descriptive spaces $K$-descriptive spaces.

(C) 1994 American Mathematical Society 
Proposition 1. Let $T$ be hereditarily Baire. Then $f$ is a PC function if and only if $f$ has a $\sigma$-scattered function base consisting of $\mathscr{F} \wedge \mathscr{G}$ sets.

We prove, moreover, the following theorem, which is a partial answer to some of natural questions on completely $\operatorname{Suslin}(\mathscr{B})$-additive families in almost $K$-descriptive or $K$-descriptive spaces (cf. [H2, p. 48; F1, p. 369]). Here $\mathscr{B}$ stands for the class of all Borel sets.

Theorem 2 (under (A)). Let $X$ be a regular Hausdorff space.

(a) Let $\left\{X_{a} \mid a \in A\right\}$ be a completely almost $K$-descriptive-additive and disjoint family in $X$. Then there is a $\sigma$-scattered network for $\left\{X_{a}\right\}$ in $X$.

(b) Let $\left\{X_{a} \mid a \in A\right\}$ be a completely $K$-descriptive-additive and disjoint family in $X$. Then there is a $\sigma$-relatively discrete network for $\left\{X_{a}\right\}$ in $X$.

Corollary. (a) Let $X$ be a completely regular almost $K$-descriptive Hausdorff space, and let $\left\{X_{a} \mid a \in A\right\}$ be a completely Suslin( $\left.\mathscr{B}_{s}\right)$-additive family in $X$. Then $\left\{X_{a} \mid a \in A\right\}$ has a $\sigma$-scattered network.

(b) Let $X$ be a $K$-descriptive regular Hausdorff space, and let $\left\{X_{a} \mid a \in A\right\}$ be a completely Suslin(F)-additive and disjoint family in $X$. Then $\left\{X_{a} \mid a \in A\right\}$ has a $\sigma$-relatively discrete network.

\section{NOTATION AND BASIC NOTIONS}

Let us first recall the notions used in the statements. We denote by $\mathscr{F}=$ $\mathscr{F}(X)$ the collection of all closed, by $\mathscr{G}=\mathscr{G}(X)$ of all open, and by $\mathscr{B}$ the collection of all Borel subsets of $X$. The symbol $\mathscr{F} \wedge \mathscr{G}$ stands for the collection of all sets of the form $F \cap G$ with $F \in \mathscr{F}$ and $G \in \mathscr{G}$, and $\mathscr{H}_{\sigma}$ stands for countable unions of elements of $\mathscr{H}$ as usual.

A family $\left\{X_{a} \mid a \in A\right\}$ is completely $\mathscr{H}$-additive if $\bigcup\left\{X_{a} \mid a \in B\right\} \in \mathscr{H}$ for every $B \subset A$.

The map $f$ of a topological space $X$ to a topological space $Y$ is called $\mathscr{H}$-measurable if preimages, under $f$, of open subsets of $Y$ are from $\mathscr{H}$.

The space $X$ is hereditarily Baire if each closed subspace is a Baire space (i.e., has the Baire category property).

A collection $\mathscr{C}$ of subsets of a topological space $X$ is called scattered if it is disjoint and there is a well-ordering $\prec$ on $\mathscr{C}$ such that $\bigcup\{C \in \mathscr{C} \mid C \prec D\}$ is open in $\bigcup \mathscr{C}$ for $D \in \mathscr{C}$. The collection is relatively discrete if it is topologically discrete in its union. The collection is $\sigma$-scattered, or $\sigma$-relatively discrete, if it is the union of countably many scattered, or relatively discrete, collections, respectively.

The class of all unions of scattered families of sets from $\mathscr{H}$ is denoted by $\mathscr{H}_{s}$.

Suslin $(\mathscr{H})$ denotes the class of all results of the Suslin operation operating on sets from $\mathscr{H}$, i.e., sets $S$ of the form

$$
S=\bigcup_{\sigma \in \mathbb{N}^{\mathbb{N}}} \bigcap_{n \in \mathbb{N}} S_{\left(\sigma_{1}, \ldots, \sigma_{n}\right)}
$$

with $S_{\sigma \mid n}=S_{\left(\sigma_{1}, \ldots, \sigma_{n}\right)} \in \mathscr{H}$. Also $\mathscr{H}_{s}$ denotes the class of all unions of scattered collections of sets from $\mathscr{H}$.

We say that $\mathscr{D}$ is a function base (or network) for $f: X \rightarrow Y$ if $\mathscr{D}$ is a base (or network) for $\mathscr{H}=\left\{f^{-1}(G) \mid G\right.$ open in $\left.Y\right\}$, i.e., $H=\bigcup\{D \in \mathscr{D} \mid D \subset H\}$ for $H \in \mathscr{H}$. 
A topological space $X$ is almost $K$-descriptive if there is a complete metric space $M$ and an upper semicontinuous compact-valued map of $M$ onto $X$ which takes discrete families of sets in $M$ to point-countable families with $\sigma$-scattered network.

$X$ is $K$-descriptive if there is an upper semicontinuous compact-valued map of a complete metric space $M$ onto $X$ which takes discrete families to pointcountable families with $\sigma$-relatively discrete network (this definition is introduced in [H2], or in [F1] under the name $W T$-analytic spaces).

Recall now the basic facts on almost $K$-descriptive and $K$-descriptive spaces that we use below.

Proposition 2. $\left(\mathrm{a}_{1}\right)$ Every almost $K$-descriptive space $X$ has the restricted Baire property in any topological embedding; i.e., $X \cap F$ has the Baire property with respect to $F$ for any subset $F$ in every topological embedding of $X$.

$\left(a_{2}\right)$ The union of any scattered family of almost $K$-descriptive subspaces of a topological space is an almost $K$-descriptive space, too.

$\left(\mathrm{a}_{3}\right)$ The completely regular Hausdorff space $X$ is almost $K$-descriptive if and only if it belongs to Suslin( $\left.\mathscr{B}_{s}\right)$ in some or every compactification. (Hence, every $C$ ech analytic space is almost $K$-descriptive.)

$\left(a_{4}\right)$ Every set from Suslin $\left(\mathscr{B}_{s}\right)$ in the completely regular Hausdorff almost $K$-descriptive space $X$ is almost $K$-descriptive as a subspace.

$\left(b_{1}\right)$ For every open cover of the $K$-descriptive space $X$ there is a $\sigma$-relatively discrete refinement covering $X$.

$\left(b_{2}\right)$ The sets from Suslin $(\mathscr{F})$ in a $K$-descriptive space are $K$-descriptive.

$\left(b_{3}\right)$ Every $K$-descriptive space is almost $K$-descriptive; thus, it has the restricted Baire property in any topological embedding due to $\left(\mathrm{a}_{1}\right)$.

(c) For every almost $K$-descriptive space, or $K$-descriptive space, there is a cardinal $\kappa$ (arbitrarily large) such that the completely metrizable space $\kappa^{\mathbb{N}}(\kappa$ taken with the discrete topology) can be used for $M$ in the parameterization from the respective definitions above.

Proof. The assertion $\left(\mathrm{a}_{1}\right)$ is proved in [H2, Theorem 4.1].

Let us prove $\left(a_{2}\right)$. Let $\left\{X_{a} \mid a \in A\right\}$ be a scattered family of almost $K$ descriptive subspaces of $X$. Then the parameterization of $\bigcup\left\{X_{a} \mid a \in A\right\}$ may be defined from the discrete sum of $M_{a}$ 's by $f(x)=f_{a}(x)$ for $x \in M_{a}$ where $f_{a}: M_{a} \rightarrow X_{a}$ are the parametrizations from the definition of almost $K$-descriptive spaces $X_{a}$ 's.

The assertion $\left(a_{3}\right)$ is proved in [Ho, Theorem $3 \mathrm{j}$.

To prove $\left(a_{4}\right)$, we use $\left(a_{3}\right)$. Thus we may suppose that $X$ is a set from $\operatorname{Suslin}\left(\mathscr{B}_{s}\right)$ in a compactification $K$. Every Borel set in $X$ is a $\operatorname{Suslin}\left(\mathscr{B}_{s}\right)$ again as the intersection of a Borel and a $\operatorname{Suslin}\left(\mathscr{B}_{S}\right)$ set in $K$. The sets relatively from $\mathscr{B}_{s}$ in $\operatorname{Suslin}\left(\mathscr{B}_{s}\right)$ subspace $X$ are in $\operatorname{Suslin}\left(\mathscr{B}_{s}\right)$ by $\left(\mathrm{a}_{2}\right)$ and $\left(\mathrm{a}_{3}\right)$. The rest follows from the well-known property of the Suslin operation that $\operatorname{Suslin}\left(\operatorname{Suslin}\left(\mathscr{B}_{s}\right)\right)$ is $\operatorname{Suslin}\left(\mathscr{B}_{s}\right)$.

The assertion $\left(b_{1}\right)$ is mentioned in [F1, p. 369] and it follows in a standard way. Let $\mathscr{U}$ be an open cover of $X$. We suppose that $f: M \rightarrow X$ is some parametrization of $X$ from the definition of $K$-descriptive spaces. Let $\mathscr{V}$ be the cover formed by all unions of finite collections of sets from $\mathscr{U}$. Then the "preimages" $\{x \in M \mid f(x) \subset V\}$ of sets $V$ from $\mathscr{V}$ form an open cover of $M$. The rest follows from the paracompactness of $M$, the properties of $f$, and the fact that $\mathscr{V}$ arose as finite unions of elements of $\mathscr{U}$. 
To prove $\left(b_{2}\right)$, we suppose that $X$ is a set from $\operatorname{Suslin}(\mathscr{F})$ in a $K$-descriptive space $Y$ with the $K$-descriptive parametrization $f: M \rightarrow Y$. It is easy to show in a standard way that also $Y \times \mathbb{N}^{\mathbb{N}}$ has a $K$-descriptive parametrization. The same holds for every closed subset of $Y \times \mathbb{N}^{\mathbb{N}}$. Since $X$ is the projection of some closed subset of $Y \times \mathbb{N}^{\mathrm{N}}$, we get the assertion according to [F1, Lemma] or [H2, Lemma 7.1].

The assertion (c) follows, for example, [FH, §3.2, Proposition], [H3, §1.2], and [Ho, Lemma 3].

\section{ProOFs}

Now we are going to prove Theorems 1 and 2. We proceed in a series of auxiliary results.

Lemma 1. (a) If a family $\left\{X_{a} \mid a \in A\right\}$ in $X$ has locally a $\sigma$-scattered network, i.e., every element of $X$ has a neighbourhood $U$ such that $\left\{U \cap X_{a}\right\}$ has a $\sigma$-scattered network, then $\left\{X_{a}\right\}$ has a $\sigma$-scattered network, too.

(b) If $\left\{X_{a} \mid a \in A\right\}$ has locally a $\sigma$-relatively discrete network and if open covers of $\bigcup\left\{X_{a}\right\}$ have $\sigma$-relatively discrete refinements covering $\bigcup\left\{X_{a}\right\}$, then $\left\{X_{a}\right\}$ has a $\sigma$-relatively discrete network (especially, the last is true if the union is $K$-descriptive).

Proof. It follows immediately since every open cover of $X$ has a scattered refinement covering $X$ and $\bigcup_{b \in B} \cup \mathscr{S}_{b}$ has a $\sigma$-scattered network if the families $\mathscr{S}_{b}$ of subsets of $X$ have $\sigma$-scattered networks and the family $\left\{\bigcup S_{b} \mid b \in B\right\}$ is $\sigma$-scattered. The similar argument works also for the other assertion. We use Proposition $2\left(b_{1}\right)$ to prove the parenthesized assertion.

Lemma 2 (under (A)). (a) If $\left\{X_{a} \mid a \in A\right\}$ is a family which has no $\sigma$-scattered network in $X$, then there are disjoint subsets $A_{0}, A_{1}$ of $A$ such that $\left\{X_{a} \mid a \in A_{i}\right\}$ has no $\sigma$-scattered network for $i=0,1$.

(b) If $\left\{X_{a} \mid a \in A\right\}$ is a family which has no $\sigma$-relatively discrete network in $X$, then there are disjoint subsets $A_{0}, A_{1}$ of $A$ such that $\left\{X_{a} \mid a \in A_{i}\right\}$ has no $\sigma$-relatively discrete network for $i=0,1$.

Proof. If it is not the case, put $\mu(B)=0$ if $\left\{X_{a} \mid a \in B\right\}$ has a $\sigma$-scattered (or $\sigma$-relatively discrete in the case (b)) network and $\mu(B)=1$ otherwise. This contradicts (A).

Lemma 3 (under (A)). (a) If $\left\{X_{a} \mid a \in A\right\}$ is a family of subsets of $X$ which has no $\sigma$-scattered network in $X$, then there are distinct elements $x_{0}$ and $x_{1}$ of $X$ and disjoint subsets $A_{0}, A_{1}$ of $A$ such that $U_{i} \cap\left\{X_{a} \mid a \in A_{i}\right\}$ has no $\sigma$-scattered network for any open neighbourhoods $U_{i}$ of $x_{i}, i=0,1$.

(b) If $\left\{X_{a} \mid a \in A\right\}$ is a completely $K$-descriptive-additive and point-countable family which has no $\sigma$-relatively discrete network in $X$, then there are distinct elements $x_{0}$ and $x_{1}$ of $X$ and disjoint subsets $A_{0}, A_{1}$ of $A$ such that $U_{i} \cap$ $\left\{X_{a} \mid a \in A_{i}\right\}$ has no $\sigma$-relatively discrete network for any open neighbourhoods $U_{i}$ of $x_{i}, i=0,1$.

Proof. We prove (a) first. We use Lemma 2 to get $A_{0}, A_{1}$. Then we can find $x_{0}$ or $x_{1}^{1}$ according to Lemma 1 so that the families $\left\{X_{a} \cap U_{i} \mid a \in A_{i}\right\}, i=0,1$, have no $\sigma$-scattered network for any neighbourhoods $U_{0}, U_{1}$ of $x_{0}, x_{1}^{1}$. To find distinct $x_{0}$ and $x_{1}$, we use Lemma 1 for the space $Y=X \backslash\left\{x_{1}^{1}\right\}$ and 
for the family $\left\{X_{a} \backslash\left\{x_{1}^{1}\right\} \mid a \in A_{1}\right\}$, which has no $\sigma$-scattered network, to find an $x_{1}^{2} \in Y$ such that the family $\left\{\left(X_{a} \backslash\left\{x_{1}^{1}\right\}\right) \cap U \mid a \in A\right\}$ has no $\sigma$-scattered network for every neighbourhood $U$ of $x_{1}^{2}$. Now $x_{1}^{1}$ and $x_{1}^{2}$ are distinct, and we can choose one of them for $x_{1}$.

We proceed similarly in the case (b) to find $x_{0}$ and $x_{1}^{1}$. Then we consider the space $Y=\bigcup\left\{X_{a} \mid a \in A, x_{1}^{1} \notin X_{a}\right\}$. Since the family $\left\{X_{a} \mid a \in A\right\}$ is completely $K$-descriptive-additive, the space $Y$ is $K$-descriptive. The family $\left\{X_{a} \mid a \in A\right\}$ is point-countable and so the family $\left\{X_{a} \mid a \in A, x_{1}^{1} \notin X_{a}\right\}$ has no $\sigma$-relatively discrete network. So we can use Lemma 1 to find $x_{1}^{2}$ in $Y$ and finish the proof like that of (a).

The main construction needed to prove Theorem 2 is contained in the following lemma. It goes back to [H3], but some modifications are needed because not only $\operatorname{Suslin}(\mathscr{F})$ sets are studied, and the family may not be countable even in a compact space.

Lemma 4 (under (A)). (a) Let $X$ be a completely regular Hausdorf space and $\left\{X_{a} \mid a \in A\right\}$ be disjoint completely almost $K$-descriptive-additive family, and let $\left\{X_{a} \mid a \in A\right\}$ have no $\sigma$-scattered network. Then there is a compact subspace $K$ of $\bigcup\left\{X_{a} \mid a \in A\right\}$ and a perfect map $\varphi$ of $K$ onto the Cantor set $C$ such that the preimages $\varphi^{-1}(l)$ of elements $l$ from $C$ are covered by families $\left\{X_{a} \mid a \in A_{l}\right\}$ where $\left\{A_{l} \mid l \in C\right\}$ forms a disjoint family of subsets of $A$.

(b) Let $X$ be a completely regular Hausdorff space and $\left\{X_{a} \mid a \in A\right\}$ be disjoint completely $K$-descriptive-additive family, and let $\left\{X_{a} \mid a \in A\right\}$ have no $\sigma$ relatively discrete network. Then there is a compact subspace $K$ of $\bigcup\left\{X_{a} \mid a \in A\right\}$ and a perfect map $\varphi$ of $K$ onto the Cantor set $C$ such that the preimages $\varphi^{-1}(l)$ of elements $l$ from $C$ are covered by families $\left\{X_{a} \mid a \in A_{l}\right\}$ where $\left\{A_{l} \mid l \in C\right\}$ forms a disjoint family of subsets of $A$.

Proof. According to Proposition 2(c) there is a $\kappa$ such that every union $X_{B}=$ $\bigcup\left\{X_{a} \mid a \in B\right\}$ for $B \subset A$ can be parametrized by an upper semicontinuous compact-valued map $f_{B}$ of the complete metric space $\kappa^{\mathrm{N}}$ onto $X_{B}$ which takes discrete families of subsets of $\kappa^{\mathbb{N}}$ to point-countable families which have a $\sigma$-scattered, or $\sigma$-relatively discrete, network, respectively.

We use the notation

$$
X_{\sigma \mid n}^{B}=f_{B}(I(\sigma \mid n))
$$

where $I(\sigma \mid n)$ stands for the set of sequences from $\kappa^{\mathbb{N}}$ which begin by $\sigma \mid n$. The spaces $X_{\sigma \mid n}^{B}$ are almost $K$-descriptive, or $K$-descriptive, respectively, because $I(\sigma \mid n)$ are closed subsets of $\kappa^{\mathbb{N}}$.

We will construct for $l \in\{0,1\}^{\mathbb{N}}$ closed sets $F_{l \mid n} \subset X$, sets $A_{l \mid n} \subset A$, finite sequences of elements of $\kappa$ called $\sigma^{i}(l \mid n), i=1, \ldots, n$, and almost $K$-descriptive, or $K$-descriptive, subspaces $Y_{l \mid n}$ such that under the convention that " $i \mid 0, i " \equiv i$ the following hold for $n=1, \ldots$.

(i) $F_{l \mid n-1,0} \cap F_{l \mid n-1,1}=\varnothing$ and, for $n \geq 2, F_{l \mid n} \subset F_{l \mid n-1}$.

(ii) $A_{l \mid n-1,0} \cap A_{l \mid n-1,1}=\varnothing$ and, for $n \geq 2, A_{l \mid n} \subset A_{l \mid n-1}$.

(iii) The length of $\sigma^{i}(l \mid n)$ is $n+1-i$ and, for $n \geq 2, \sigma^{i}(l \mid n)$ extends $\sigma^{i}(l \mid n-1)$.

(iv) $Y_{l \mid n}=F_{l \mid n} \cap X_{\sigma^{n}(l \mid n)}^{A_{l \mid n}} \cap \cdots \cap X_{\sigma^{1}(l \mid n)}^{A_{l \mid 1}}$. 
(v) $\left\{Y_{l \mid n} \cap X_{a} \mid a \in A_{l \mid n}\right\}$ has no $\sigma$-scattered, or $\sigma$-relatively discrete, network, respectively.

We proceed by induction. Since the first step is almost identical with the induction one, we suppose that $F_{l \mid k}, A_{l \mid k}, \sigma^{i}(l \mid k), i=1, \ldots, k$, and thus also $Y_{l \mid k}$ have been already chosen for $k=0,1, \ldots, n$ so that (i)-(v) hold. Let us choose an arbitrary sequence $l \mid n \in\{0,1\}^{n}$, and we find $F_{l \mid n, j}, A_{\imath \mid n, j}$, $\sigma^{i}(l \mid n, j), i=1, \ldots, n+1$, such that $(\mathrm{i})-(\mathrm{v})$ be satisfied for them, too. Since according to $(\mathrm{v})$ the collection $\left\{Y_{l \mid n} \cap X_{a} \mid a \in A_{l \mid n}\right\}$ has no $\sigma$-scattered network, or no $\sigma$-relatively discrete network, respectively, we can use Lemma 3 to find $x_{0}$ and $x_{1}, A_{l \mid n, 0}$ and $A_{l \mid n, 1}$, and the closed neighbourhoods $F_{i \mid n, 0}$ and $F_{i \mid n, 1}$ of $x_{0}$ and $x_{1}$ such that (i) and (ii) are fulfilled and so that

$$
\left\{X_{a} \cap Y_{l \mid n} \cap F_{l \mid n, j} \mid a \in A_{l \mid n, j}\right\}
$$

has no $\sigma$-scattered (or $\sigma$-relatively discrete in the case (b)) network because the space $X$ is Hausdorff, and, moreover, $\left\{X_{a} \cap Y_{l \mid n}\right\}$ are completely $K$-descriptiveadditive in the case (b). The set $Y_{l \mid n} \cap F_{l \mid n, j}$ is covered by the sets

$$
X_{j_{n+1}}^{A_{l \mid n+1}} \cap \cdots \cap X_{\sigma^{1}(l \mid n), j_{1}}^{A_{t_{1}}} \cap F_{l \mid n+1}
$$

with $j_{1}, \ldots, j_{n+1}$ from $\kappa$ and $l_{n+1}=j$, which have a $\sigma$-scattered, or $\sigma$ relatively discrete, network, respectively.

We choose $j_{1}, \ldots, j_{n+1} \in \kappa$ so that (iv) is fulfilled with $\sigma^{1}(l \mid n+1)=$ $\left(\sigma^{1}(l \mid n), j_{1}\right), \ldots, \sigma^{n+1}(l \mid n+1)=j_{n+1}$.

Put

$$
K=\bigcup_{l \in \mathcal{K}^{\mathrm{N}}} \bigcap_{n \in \mathbb{N}} \overline{Y_{l \mid n}}=\bigcap_{n \in \mathbb{N}} \bigcup_{l \mid n \in \mathcal{K}^{n}} \overline{Y_{l \mid n}}
$$

The second equality holds due to (i) and $K$ is closed in $X$.

According to (iii) for every $l \in\{0,1\}^{\mathbb{N}}$ there is a limit $\sigma^{i}(l)=\lim _{n \rightarrow \infty} \sigma^{i}(l \mid n)$ $\in \kappa^{\mathrm{N}}$.

Since the parametrizations $f_{A_{|| n}}$ are upper semicontinuous and compactvalued, and due to (iv), we get that there is an $m \in \mathbb{N}$ such that

$$
Y_{l \mid(m+n-1)} \subset X_{\sigma^{n}(l \mid m)}^{A_{i \mid n}}=f_{A_{i \mid n}}\left(I\left(\sigma^{n}(l) \mid m\right)\right) \subset G
$$

for every open set $G$ containing $f_{A_{l \mid n}}\left(\sigma^{n}(l)\right)$.

Because $X$ is regular, we can find $m$ such that $\overline{Y_{\imath \mid m}} \subset G$. It follows easily that

$$
K(l)=\bigcap \overline{Y_{l \mid n}} \subset X_{\sigma^{m}(l)}^{A_{l \mid m}} \subset X^{A_{l \mid m}}
$$

for $m \in \mathbb{N}$.

In particular, the intersections $K(l)$ are contained in the compact set

$$
f_{A_{0}}\left(\left\{\sigma^{1}\left(0, l_{2}, \ldots\right) \mid l \in\{0,1\}^{\mathbb{N}}\right\}\right) \cup f_{A_{1}}\left(\left\{\sigma^{1}\left(1, l_{2}, \ldots\right) \mid l \in\{0,1\}^{\mathbb{N}}\right\}\right) .
$$

It is compact because $f_{A_{i \mid n}}$ are upper semicontinuous and compact-valued and the sets $\left\{\sigma^{1}\left(i, l_{2}, \ldots\right) \mid l \in\{0,1\}^{N}\right\}, i=0,1$, are compact.

Thus $K$, as a closed subset of a compact set, is compact.

According to $(\mathrm{v})$ it is $Y_{l \mid n} \neq \varnothing$. Since the sets $F_{l \mid n}$ are closed, and due to (iv) and $(*),(* *)$, the intersections $K(l)$ are nonempty. 
Now we can note that the map $\varphi: K \rightarrow 2^{\mathbb{N}}$ defined by $l$ on $K(l)$ is continuous because $\overline{Y(l \mid n)} \cap K$ is open in $K$ and $\overline{Y(l \mid n)} \cap \overline{Y\left(l^{\prime} \mid n\right)}=\varnothing$ for $\imath^{\prime}|n \neq l| n$ by (i).

Define $A_{l}=\left\{a \in A \mid X_{a} \cap K(l) \neq \varnothing\right\}$. We get the last assertion of Lemma 4. If $a \in A_{l}$ and $l^{\prime} \neq l$, then there is an $m \in \mathbb{N}$ such that $l\left|m \neq l^{\prime}\right| m$. Then $a \notin A_{l^{\prime}}$ because it would be $\varnothing \neq X_{a} \cap K\left(l^{\prime}\right) \subset X^{A_{t^{\prime} \mid m}}$ according to (**) otherwise. But $X_{a} \subset X^{A_{i \mid m}}$ and $A_{l \mid m} \cap A_{l^{\prime} \mid m}=\varnothing$ due to (ii).

Remark. Our original idea was to use now the map $\varphi$ constructed in Lemma 5 and the following result of Frolik [F2].

Proposition. Let $X$ be a Čech analytic space (i.e., a Suslin $\mathscr{B})$ subset of some compactification) and $\varphi$ a perfect map of $X$ onto a metric space $M$. Then $M$ is Suslin $(\mathscr{F})$ in its completion.

To prove Theorem 1 for completely Čech analytic-additive families, we should realize that for any subset $D$ of $C$ the map $\varphi \uparrow \varphi^{-1}(D): \varphi^{-1}(D) \rightarrow D$ is perfect, and taking a nonanalytic subset of $C$ for $D$, we get a contradiction.

It would work for $\operatorname{Suslin}(\mathscr{B})$-additive families only. Hensell drew our attention to $[\mathrm{P}]$, and we use the idea from there to get our result for almost $K$-descriptive additivity. Instead of the mentioned Frolik's result we use the following observation from [S] used in [P] analogously.

Lemma 5. Let $f: K \rightarrow C$ be a continuous map of the compact space $K$ onto $C$ such that $f^{-1}(A)$ has the restricted Baire property. Then $A$ has the Baire property.

Proof of Theorem 2 and Corollary. Let $\varphi: K \rightarrow C$ be the map from Lemma 4. Then the preimage of any subset $S$ of $C$ is necessarily almost $K$-descriptive (by Proposition $2\left(b_{3}\right)$ in the case $\left.(b)\right)$, and by Proposition $2\left(a_{1}\right)$ it has the restricted Baire property in $K$, and thus $S$ has the Baire property due to Lemma 5 . This is impossible by [K, 11, IVa].

The corollary follows by Proposition $2\left(a_{3}\right)$ and $\left(b_{2}\right)$.

To get Theorem 1 we use Propositions 1 and 2, Theorem 2, and

Lemma 6. If a point-countable family $\left\{X_{a} \mid a \in A\right\}$ consisting of sets from $(\mathscr{F} \wedge \mathscr{G})_{\sigma}$ has a $\sigma$-scattered network then the network may be chosen to consist of sets from $\mathscr{F} \wedge \mathscr{G}$.

Proof. Let $\mathscr{N}=\bigcup_{n \in \mathbb{N}} \mathscr{N}_{n}$ with $\mathscr{N}_{n}$ scattered is the network for $\left\{X_{a}\right\}$. Due to [H2, Lemma 2.3] there are scattered collections $\mathscr{B}_{n}=\left\{B(N) \supset N \mid N \in \mathscr{N}_{n}\right\}$ of $(\mathscr{F} \wedge \mathscr{G})$-sets. Let $X_{a}=\bigcup X_{a m}$ with $X_{a m}$ being from $\mathscr{F} \wedge \mathscr{G}$. We put

$$
\mathscr{C}_{n, m}=\left\{X_{a m} \cap B(N) \mid a \in A \text { with } N \subset X_{a} \text { and } N \in \mathscr{N}_{n}\right\} \text {. }
$$

These families are $\sigma$-scattered since $N$ is contained in at most countably many $X_{a}$ 's and $\bigcup_{n, m} \mathscr{C}_{n, m}$ is a network since $\mathscr{N}$ was.

Proof of Theorem 1. It runs in a standard way. Let $\mathscr{U}=U_{n} \mathscr{U}_{n}$ be an open base in the metric space $X$ with all $\mathscr{U}_{n}$ discrete. Then $\left\{f^{-1}(U) \mid U \in \mathscr{U}_{n}\right\}$, $n=1,2, \ldots$, form completely $(\mathscr{F} \wedge \mathscr{G})_{\sigma}$-additive disjoint families in the almost $K$-descriptive space $T$. Thus, due to Theorem 2 and Lemma 6 , they have $\sigma$-scattered networks, say $\mathscr{N}_{n}$, consisting of $(\mathscr{F} \wedge \mathscr{G})$-sets and $\bigcup_{n} \mathscr{N}_{n}$ is 
therefore a $\sigma$-scattered base for $f$. We use Proposition 1 to finish the proof of Theorem 1.

Remarks. It is proved in [H1, Theorem 2.5] that a $P C$-function $f: T \rightarrow X$ is $(\mathscr{F} \wedge \mathscr{G})_{\sigma}$-measurable if and only if every scattered partition of $T$ is completely $(\mathscr{F} \wedge \mathscr{G})_{\sigma}$-additive.

We left open the following problems:

Do Theorems 1 and 2 hold independently on the additional axiom (A)?

Does Theorem 2 hold for point-finite families?

Is it possible to find a $\sigma$-relatively discrete network for a completely $\operatorname{Suslin}(\mathscr{B})$-additive, disjoint, or point-finite family in a Čech analytic space? This is a question from [H2, p. 48].

\section{ACKNOWLEDGMENT}

I would like to thank R. W. Hansell, J. Pelant, and L. Zajíček for helpful remarks and discussions.

\section{REFERENCES}

[F1] Z. Frolik, Distinguished subclasses of Čech-analytic spaces, Comment. Math. Univ. Carolin. 25 (1984), 368-370.

[F2] _ Refinements of perfect maps onto metric spaces and an application to Čech-analytic spaces, Topology Appl. 33 (1989), 77-84.

[FH] Z. Frolik and P. Holický, Analytic and Luzin spaces (non-separable case), Topology Appl. 19 (1985), 129-156.

[H1] R. W. Hansell, First class functions with values in nonseparable spaces, Constantin Carathéodory: An International Tribute, Vols. I, II, World Sci. Publishing, Teaneck, NJ, 1991, pp. 461-475.

[H2] _ Descriptive sets and the topology of nonseparable Banach spaces, preprint.

[H3] _ Borel measurable mappings for nonseparable metric spaces, Trans. Amer. Math. Soc. 161 (1971), 145-169.

[Ho] P. Holický, Čech analytic and almost $K$-descriptive spaces, Czechoslovak Math. J. (to appear).

[JNR] J. E. Jayne, I. Namioka, and C. A. Rogers, Properties like the Radon-Nikodým property, preprint.

[K] K. Kuratowski, Topology, vol. 1, PWN, Warsaw, 1966.

[P] R. Pol, Remark on the restricted Baire property in compact spaces, Bull. Acad. Polon Sci. 24 (1976), 599-603.

[S] Z. Semadeni, Banach spaces of continuous functions, PWN, Warsaw, 1971.

Department of Mathematical Analysis, Charles University, Sokolovska 83, 18600 Praha, Czech Republic

E-mail address: holicky@CSPGUK11. BITNET 